\title{
REVIEW \\ Spinal cord injury models: a review
}

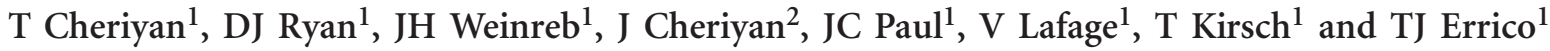

Background: Animal spinal cord injury ( $\mathrm{SCl}$ ) models have proved invaluable in better understanding the mechanisms involved in traumatic $\mathrm{SCl}$ and evaluating the effectiveness of experimental therapeutic interventions. Over the past 25 years, substantial gains have been made in developing consistent, reproducible and reliable animal SCI models.

Study design: Review.

Objective: The objective of this review was to consolidate current knowledge on SCl models and introduce newer paradigms that are currently being developed.

Results: SCl models are categorized based on the mechanism of injury into contusion, compression, distraction, dislocation, transection or chemical models. Contusion devices inflict a transient, acute injury to the spinal cord using a weight-drop technique, electromagnetic impactor or air pressure. Compression devices compress the cord at specific force and duration to cause SCl. Distraction SCl devices inflict graded injury by controlled stretching of the cord. Mechanical displacement of the vertebrae is utilized to produce dislocation-type SCI. Surgical transection of the cord, partial or complete, is particularly useful in regenerative medicine. Finally, chemically induced SCI replicates select components of the secondary injury cascade. Although rodents remain the most commonly used species and are best suited for preliminary SCl studies, large animal and nonhuman primate experiments better approximate human SCI.

Conclusion: All SCl models aim to replicate $\mathrm{SCl}$ in humans as closely as possible. Given the recent improvements in commonly used models and development of newer paradigms, much progress is anticipated in the coming years.

Spinal Cord (2014) 52, 588-595; doi:10.1038/sc.2014.91; published online 10 June 2014

\section{INTRODUCTION}

Spinal cord injury (SCI) models have proved indispensible not only for investigating the efficacy of therapeutic interventions but also for better understanding the molecular pathways involved. SCI models have evolved significantly over the past century since Allen developed the first weight-drop contusion model in $1911 .^{1}$ SCI models aim to recreate features of human SCI as closely as possible. These models vary in terms of the animal utilized, site of injury infliction and injury mechanism.

Rats are used most commonly in preliminary studies as they are relatively inexpensive, readily available and have demonstrated similar functional, electrophysiological, and morphological outcomes to humans following SCI. ${ }^{2}$ Mice are particularly useful for genetic studies. ${ }^{3}$ Nonhuman primate SCI models_including marmosets, macaques and squirrel monkeys-better approximate human SCI than rodent models, and they accommodate assessment of multiple recovery variables and rehabilitative therapy. ${ }^{4}$ New world primates like marmosets confer advantages over old world primates as they are smaller, easier to handle, have a higher breeding efficiency and can be bred in experimental colonies. SCI models that incorporate large animals—such as pigs or dogs—can also be used when it is important for further validation of experiments.

Though most studies have induced a thoracic-level injury, recent research has also focused on cervical-level injury models because of their clinical relevance. ${ }^{5}$ Human SCIs commonly occur at the cervical level, especially following nonpenetrating trauma such as sport injury and motor vehicle accident. These models result in distinct neural deficits than thoracic injuries and should be considered in terms of therapeutic approaches. ${ }^{5}$ Lumbar-level SCI models have also been described, although less commonly. ${ }^{6}$

Based on the mechanism of injury, SCI models can be classified as contusion, compression, distraction, dislocation, transection or chemical. Contusion models, in which a transient force is applied to displace and damage the spinal cord, include weight-drop, electromagnetic and air pressure devices. Compression models are characterized by compression of the spinal cord over an extended period of time. Distraction models apply opposing traction forces to stretch the spinal cord, whereas dislocation models inflict injury by lateral displacement of vertebra. Transection involves partial or complete severing of the spinal cord at a particular level. Finally, chemicalmediated SCI models investigate specific aspects of the secondary damage sequence. This review provides an overview of current in vivo animal SCI models and recent developments in the field.

\section{CONTUSION MODELS}

Contusion devices are designed to inflict a transient, acute injury to the spinal cord. The category includes weight-drop apparatuses,

${ }^{1}$ Department of Orthopaedic Surgery, Hospital for Joint Diseases, New York Langone Medical Center, New York, NY, USA and ${ }^{2}$ Department of General Surgery, University of Texas Health Science Center at San Antonio, San Antonio, TX, USA

Correspondence: Dr T Cheriyan, Spine Research Institute, 306 East, 15th Street, New York, NY 1003, USA.

E-mail: thomascheriyan@gmail.com

Received 8 September 2013; revised 23 April 2014; accepted 5 May 2014; published online 10 June 2014 
a

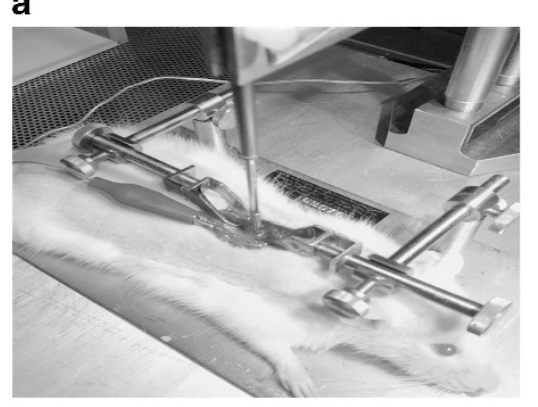

b

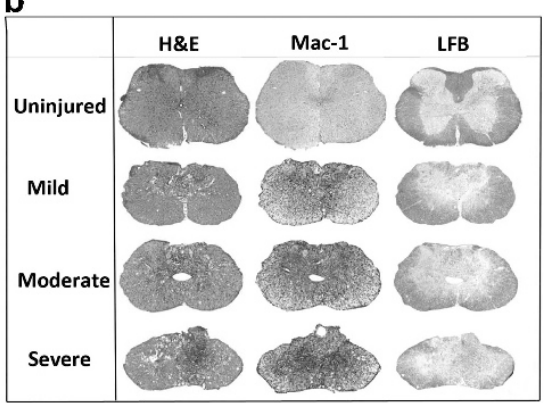

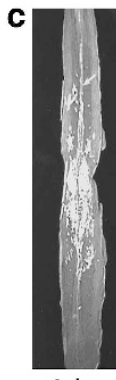

1 day

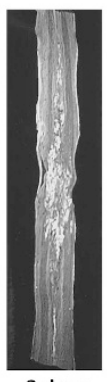

3 days

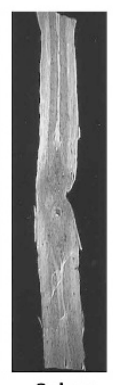

8 days

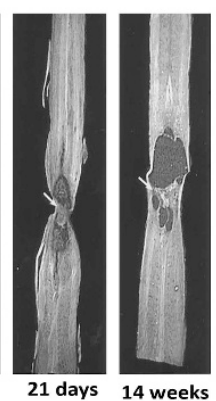

Figure 1 (a) Photograph of infliction of a contusion-type SCI on a rat model using MASCIS Impactor. After laminectomy is performed at selected level, the spinal column is stabilized using a clamp system and an impactor is used to apply injury of desired severity. (b) Representative photomicrographs of crosssection of spinal cord stained with hematoxylin and eosin, Mac-1 immunohistochemisty and luxol blue stain at 28 days after injury. With increasing severity of injury, there is evidently increasing cell damage, macrophage/microglial reactivity and myelin loss (reprinted with permission from Ghasemlou et al. Tissue displacement and impact force are important contributors to outcome after spinal cord contusion injury. Exp Neurol 196, 9-17; 2005) (c) Progressive gross histological changes seen after spinal cord injury at days $1,3,8$ and 21 , and 14 weeks. At day 1 , hemorrhage appears and extends from the epicenter rostrally. Injury area becomes progressively more defined with the formation of a cavity and cyst with time (reprinted with permission from Hill CE et al. Degeneration and sprouting of identified descending supraspinal axons after contusive spinal cord injury in rat. Exp Neurol 171, 153169; 2011).

electromagnetic impactors and a recently described air gun device (Figure 1).

\section{New York University (NYU)/Multicenter Animal Spinal Cord Injury Study (MASCIS) impactor}

The NYU impactor was first developed and described by Gruner in 1992. ${ }^{7}$ The injury procedure involves performing a laminectomy at the targeted level and stabilization of the spinal cord by means of clamps. A rod of specific weight, usually $10 \mathrm{~g}$, is dropped from a precise height above the surface of the cord to induce SCI of defined severity. Key parameters such as height, time, velocity upon impact and cord compression are recorded digitally that enable experiments to be analyzed and discarded if out of desired range.

The NYU impactor was adopted for use in the MASCIS, and renamed the MASCIS impactor. This study group recommended standardization of conditions that included the use of Long Evans rats that are $77 \pm 1$ days old, a pentobarbital dosage of 45 and $60 \mathrm{mg} \mathrm{kg}^{-1}$ for females and males, respectively, and infliction of injury $60 \pm 1 \mathrm{~min}$ after induction of anesthesia. ${ }^{8}$ Because of practical considerations, however, strict adherence to these recommendations is challenging.

Two newer versions of the MASCIS impactor have been introduced. The MASCIS II incorporates an electromagnetic push-button weight release mechanism. However, the MASCIS II does not interface with the computer, and hence digital recording of parameters is not possible. The MASCIS III, introduced in 2012, combines aspects of the two prior iterations of the device, including digital data recording and electromagnetic weight release. The improved vertebral clamping system of the MASCIS III also helps minimize movements.
The MASCIS impactor was originally described using rats, but has been modified for use in mice and marmosets. ${ }^{4,8}$ Although most of the literature involves thoracic-level injuries, the MASCIS impactor has been used to generate injury in the cervical spine in a rat model. ${ }^{5}$ Weight-drop methods in the pig and dog that rely on similar principles as the MASCIS devices have recently been described. ${ }^{9,10}$

The MASCIS impactor is widely used and produces a validated and reproducible contusion injury in rat models. However, variability due to bouncing of the impactor rod on the spinal cord after initial drop 'weight bounce', resulting in multiple impacts, has been of concern. Furthermore, duration of impact is not precisely controlled. As with all mechanical models, it is challenging to accurately control biomechanics to produce consistent and reproducible SCI.

\section{Infinite horizon (IH) impactor}

The IH device utilizes a force-controlled impact instead of weightdrop height or tissue displacement to inflict injury. A stepping motor interfaces with an external computer to apply a controlled impact. After laminectomy at desired injury site, a metal impounder inflicts injury, and an attached sensor directly measures the force between the impactor and the spinal cord tissue. This minimizes error introduced by specimen movement. When the predetermined force threshold is reached, the tip is automatically and immediately withdrawn, and the exact force transmitted is displayed. Thus, weight bounce phenomenon is not encountered.

Three different injury severities (100, 150 and $200 \mathrm{kdyn}$ ) in the rat, inflicting injury at the T10 level, were first described. ${ }^{11}$ The IH impactor has also been utilized to generate SCI in the cervical spine, 
and the device can be modified for usage with smaller rodents such as mice by simply replacing the impacting tip with a smaller one. ${ }^{12}$

A limitation observed is the variability in firmly holding the spinal column using the clamps provided with the IH device, and some researchers have developed custom-built clamping systems to overcome this. ${ }^{13}$ Although subtle movements should not theoretically influence injury mechanics of the force-controlled impactor, inconsistent parenchymal injury and functional deficits do occur.

\section{Ohio State University (OSU) impactor}

Originally developed in 1987 in the rat model, the OSU impactor was refined in 1992 to improve injury reproducibility. ${ }^{14}$ The procedure involves laminectomy at the target level and spine stabilization using clamping. Similar to the IH impactor, the OSU device uses a computer feedback-controlled electromagnetic impactor. When triggered, a probe contacts and displaces the cord $30 \mu \mathrm{m}$ to provide a replicable starting point. Then, the instrument compresses the cord a predetermined distance, generally between 0.8 and $1.1 \mathrm{~mm}$, for a set amount of time, $\sim 4$ to $5 \mathrm{~ms}$. The OSU device avoids multiple strikes, as the impounder is actively withdrawn.

The OSU impactor measures force transmitted to the spinal cord and cord displacement, which allows for exclusion of suboptimal injuries. The device was modified in 2000 to study SCI in a mouse model. ${ }^{3}$ As with the MASCIS and IH devices, the OSU impactor has recently been used to induce injury in the cervical spine of the rat. ${ }^{15}$ A limitation of the device is that it is sometimes challenging to determine the zero distance for the impactor as the spinal cord and cerebrospinal fluid is displaced slightly when initially loaded.

\section{Air gun impactor}

The air gun impactor is a new contusion device that was developed in 2012 as a rat model. ${ }^{16}$ It produces SCI using a precisely directed highpressure stream of air. The T10 vertebra is stabilized by affixing clamps to the adjacent vertebrae. A dental drill is then used to make a $2 \mathrm{~mm}$ diameter hole in the vertebral arch of target vertebra, through which the injector is inserted and positioned so that it touches, but does not put pressure on, the dura. The air 'shot' is then delivered. The pilot study created three experimental groups using $0.1 \mathrm{~s}$ shots of three different pressures, but the device also allows for a range of exposure times (up to $15 \mathrm{~min}$ ).

The results of SCI were analyzed using magnetic resonance imaging, histology and multiple behavioral assessments. For the most part, graded injury was successfully produced. However, one notable exception was in the locomotor assessment: the differences between the moderate injury and severe injury groups and the control and mild injury groups were not statistically significant. Lack of precise injury quantification will require further validation on the reliability, and reproducibility of the model.

\section{COMPRESSION MODELS}

Compression models are distinct from contusion models in that they involve prolonged cord compression. Some of these models are, in fact, contusion-compression models that involve an acute impact followed by persisting compression of the cord. This mechanism is seen in SCI because of fracture dislocations and burst fractures.

\section{Clip compression}

The use of a modified aneurysm clip (Figure 2A) to generate SCI was first described in rats in $1978 .{ }^{17}$ The procedure involves performing a laminectomy at the desired level of the spine. The clip is closed at specific force around the spinal cord, producing an acute injury, and is then left to compress the cord for a select amount of time, usually for at least $1 \mathrm{~min}$, to produce an injury. Clips with a wide range of closing force are available, generating varying closure forces and therefore injury severities in the animal. A force of 50 and $35 \mathrm{~g}$ is used to produce a severe and moderate injury, respectively. The duration of clip application can also be varied to produce injuries of varying severity.

In rats, it has been shown to produce graded injuries in the thoracic spine in terms of both functional and histological outcomes. ${ }^{18}$ In addition, the model has been used in the cervical and lumbar spine of the rat and also been adapted for use in the mouse. ${ }^{19}$ In the murine model, a smaller aneurysm clip was utilized to produce injury in the thoracic spine that varied in grade with respect to both behavioral and histological outcomes. ${ }^{20}$ Use of a modified vascular clip in the mouse was also recently shown to be effective. ${ }^{21} \mathrm{~A}$ spring-loaded clip has also been utilized to induce injury in the thoracic spine of pigs. ${ }^{22}$

The advantages of clip compression are that it provides a compression-contusion model that is relatively inexpensive, generates SCI of varying severity and is adaptable for use in all regions of the spine. Although simplicity allows for adaptability, the only injury parameters that are obtained are the calibrated closing force of the clip and the duration of compression. Thus, velocity of the clip upon contact with the cord, actual force delivered and extent of cord compression are not accounted for.

A clip compression technique can also be used to occlude the blood supply to a specific cord segment for a specific length of time to inflict ischemia/reperfusion-type SCI. Clinically, the model approximates iatrogenic SCI that may occur during surgeries like those on the thoracic aorta. The model can also be created using a balloon inflation technique and direct ligation of the vessel. ${ }^{23,24}$

\section{Calibrated forceps compression}

Forceps compression was developed to circumvent some of the biomechanical issues associated with contusion models to produce a reliable model. The use of calibrated forceps (Figure 2B) to deliver SCI was first described in 1991 in the guinea pig. ${ }^{25}$ Following laminectomy at the T13 level, a pair of forceps is compressed around the spinal cord to a specific width, using a spacer, for a select duration. A similar method has been used in rats, also in the lower thoracic spine, and injuries of a range of severities were inflicted by using different compression widths. The technique was adapted for use in mice, and has shown to be capable of producing graded SCI. ${ }^{26}$

Although forceps technique is a simple and inexpensive SCI model, it lacks the acute impact component that clip compression and contusion paradigms possess. Forceps compression therefore does not simulate an important aspect of the most common human SCI mechanisms and this makes it much less common than other injury models.

\section{Balloon compression}

This method was first described in 1953 in dogs. ${ }^{27}$ Generally, balloon compression involves the insertion of a catheter with a small, inflatable balloon affixed to its end (Figure 2C) into the epidural or subdural space. ${ }^{28}$ The balloon is then filled with saline or air to a fixed volume that compresses the spinal cord. The balloon is deflated after a specific duration. Preparation may involve laminectomy at the desired lesion site, or merely laminotomy at a location caudal to this site. Injury can be graded by varying the inflation volume, duration of compression or both.

Though rat models have been used extensively, the procedure has been refined in dogs, rabbits and primates. ${ }^{29-31}$ The advantages of the 


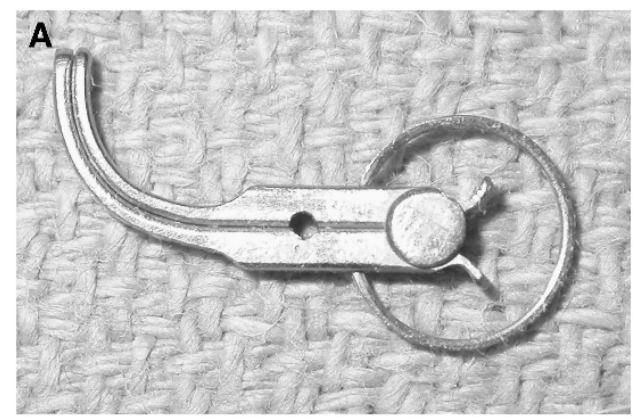

B

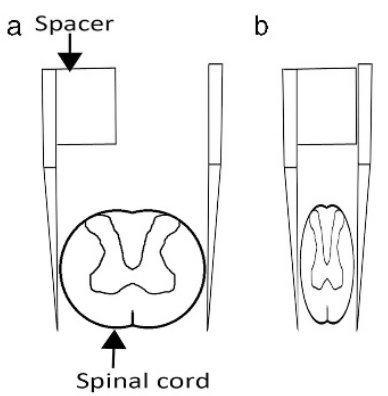

D

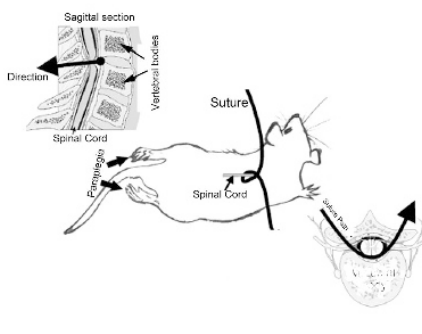

Figure 2 (A) Photograph of modified aneurysm clip used for spinal cord injury clip-compression model (courtesy: Charles Tator, University of Toronto, Toronto Western Hospital, Canada). After laminectomy at the desired level of the spine, the clip is closed at specific force around the spinal cord to produce an acute injury. The force of clip closure and duration of application can be varied to produce injury of varying severity. (B) Schematic diagram of calibrated compression forceps with spacer. Following laminectomy, a pair of forceps is compressed around the spinal cord to a specific width, using a spacer, for a select duration to produce spinal cord injury. The width and duration of compression can be modified to inflict to generate desired injury severity. (C) Balloon compression involves the insertion of a catheter with a small, inflatable balloon affixed to its end passed through a laminectomy or laminotomy caudal to the injury site. Injury can be graded by varying the inflation volume, duration of compression or both. (D) Schematic diagram of spinal cord strapping technique. A curved needle is inserted through the intervertebral foramen and the epidural space to encircle the spinal cord anteriorly and then the needle is inserted through the contralateral intervertebral foramen and back to the skin to wrap the suture around the cord. One end of the suture is affixed to the wall of the experimental device and the other is attached to a suspended mass via a simple pulley. When the weight is dropped, compression is initiated, and allowed to continue for an extended period of time (reprinted with permission from da Costa et al. ${ }^{32}$ ).

model are that it is relatively inexpensive and requires minimal surgical preparation. However, like the forceps compression model, balloon compression typically lacks the acute component of SCI. Rapid inflation of the balloon may add some level of this type of trauma; however, with no monitoring of velocity or force delivered, it would be difficult to standardize the initial impact. The balloon compression model is less commonly used than the contusion or clip compression models.

\section{Spinal cord strapping}

Spinal cord strapping is a novel SCI technique that uses a mechanical device that the developers have termed SC-STRAPPER(Figure 2D). ${ }^{32}$ The procedure involves the insertion of a curved surgical needle with suture attached into one side of the animal. The suture is then wrapped around a particular level of spinal cord in the epidural space and brought back through the skin on the other side of the rat. One end of the suture is affixed to the wall of the experimental device and the other is attached to a suspended mass via a simple pulley. When the mass is allowed to drop, compression is initiated, and it is allowed to continue for $1 \mathrm{~min}$. The investigators utilized three different masses to inflict mild, moderate and severe injuries in the rats. Results showed the injuries to be graded in terms of both functional and histological outcomes. ${ }^{32}$

The technique is advantageous in that it is a comparatively noninvasive method that does not require laminectomy or cause bleeding, and it has reported a $100 \%$ survival rate. ${ }^{32}$ The device also provides compression on all sides of the cord, not just in the dorsoventral or lateral direction, and is capable of producing graded injury pattern.

However, the consistent reproducibility of injury at precise site and extend are still to be proven. Although the force of compression can be estimated based on the weight of the mass, other parameters related to the injury can neither be measured nor controlled.

\section{DISTRACTION MODELS}

Distraction models of SCI involve controlled stretching of the cord aiming to simulate the tension forces experienced by the cord in SCI. ${ }^{33}$ Injuries of this type have been investigated in cats, dogs, monkeys and pigs. However, sophisticated and reproducible modeling of this process has been developed only recently and is an area of active research.

\section{Harrington distractor}

In 2004, Dabney et al. ${ }^{34}$ detailed the use of this device to inflict thoracic SCI in rats. The procedure involves laminectomy at the intended injury location, followed by the placement of modified Harrington hooks under the laminae of the vertebrae one level caudal and one level rostral to the injury target. These hooks are connected to a computer-controlled stepping motor that is used to control the length, speed and duration of distraction. Distraction length of 3, 5 and $7 \mathrm{~mm}$ produced mild, moderate and severe SCI when the facet capsular ligament was severed. However, a major limitation in this 
model was the high variability within each injury grade. Furthermore, distraction speed was limited to $1 \mathrm{~cm} \mathrm{~s}^{-1}$ that may be too slow to simulate human injuries. Thus, although the Harrington distractor demonstrated the promise of distraction SCI models, it has not been validated.

\section{University of British Columbia (UBC) multimechanism device}

This novel injury device, which was developed in the rat, is highly versatile; it can inflict contusion, distraction and dislocation injury (Figure 3a). ${ }^{35}$ The device is capable of providing feedback on cord displacement or force transmitted following injury. The UBC device uses clamps that wedge beneath the transverse processes against the lateral masses of the vertebrae with firm support, allowing researchers to accelerate the device components to high velocities (up to $1 \mathrm{~m} \mathrm{~s}^{-1}$ ) without vertebral slippage during injury.

Aside from clamping, the UBC device's contusion technique is similar to that implemented by the OSU and IH impactors. For distraction, C3 and C4 are jointly clamped and stabilized while another clamp holding C5 and C6 is accelerated caudally, held stationary for $1 \mathrm{~s}$ at maximal displacement, and returned to its initial position. Finally, for a dislocation injury, an identical clamping arrangement is used, but the clamp holding C5 and C6 is accelerated dorsally instead of caudally to generate SCI. The investigators were able to inflict moderate and severe SCI by manipulating displacement as the independent variable.
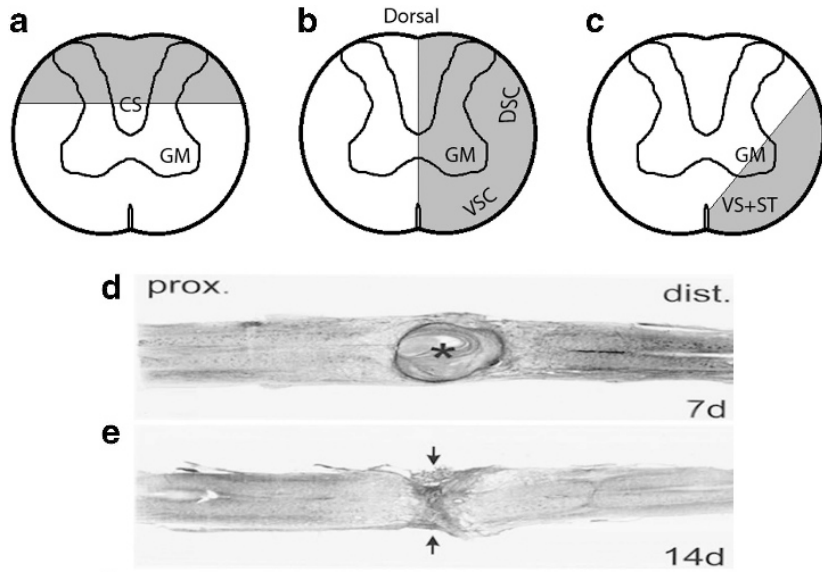

f

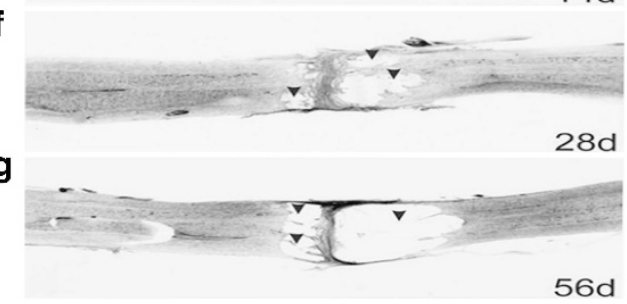

Figure 3 Schematic diagram of partial transaction of the spinal cord through (a) the ventral horn, part of the grey matter (GM) and corticospinal (CS) tract bilaterally; (b) unilateral ventral and dorsal spinal cord (VSC and DSC) and (c) unilaterally through the vestibulospinal (VS) and spinothalamic (ST) tract. ( $\mathbf{d}-\mathbf{g})$ Progressive gross longitudinal histological changes at complete transection site in rat spinal cord at 7, 14, 28 and 56 days after injury. At 7 days after injury, there is clot (asterisk) formation that gets replaced by scar tissue by day 14 (arrows). This is followed by formation of cavity (arrowheads) by 28 and 56 days (reprinted with permission from Winter et al. Injury-induced class 3 sematphorin expression in the rat spinal cord. Exp Neurol 175: 61-75; 2002).
The UBC device is capable of inflicting high-speed distraction SCI, and creates an injury that simulates vertebral dislocation. However, further validation studies including locomotor studies are necessary.

\section{University of Texas at Arlington (UTA) distractor}

The UTA distractor (Figure 3b) ${ }^{36}$ aimed to inflict a bidirectional distraction SCI as compared with the unidirectional injury generated by the UBC device. The UTA distractor used custom clips that are affixed securely to the vertebral body. These clamps are attached to linear actuators that simultaneously distract two vertebrae (T9 and T11) caudorostrally. It is relatively noninvasive, as a laminectomy is not performed, and computer software is able to record the force delivered to the spinal cord during the injury. ${ }^{36}$

Mild, moderate and severe injury was assessed based on a distance distraction of 3, 5 and $7 \mathrm{~mm}$, respectively. They used electrophysiological, behavioral and histological outcomes to measure the grade of injury. A limitation of the device was the high variability and inconsistency in behavioral scoring. The UTA distractor also inflicts SCI at much lower speed $\left(1 \mathrm{~mm} \mathrm{~s}^{-1}\right)$ than the UBC device that may affect the extent to which it mimics human injury. The device is not commercially available and a newer, more advanced version of the UTA is being currently developed by the inventors.

\section{DISLOCATION MODEL}

Fiford et al. ${ }^{37}$ developed a SCI device in rats that utilized a vertebral displacement mechanism to replicate column dislocation commonly seen in traumatic human SCI. The device consisted of two horizontal beams that can be attached the lateral surfaces of bodies of adjacent vertebrae by means of adjustable sliding clamps. One beam is rigidly fixed, whereas the other beam is used to laterally displace the lower attached vertebrae to a specified distance $(1-20 \mathrm{~mm})$ and speed $\left(50-150 \mathrm{~mm} \mathrm{~s}^{-1}\right)$ for a time period of $1 \mathrm{~s}$. The investigators showed larger displacement resulted in greater degree of injury histologically.

Choo et al..$^{35}$ described a similar procedure to produce an anterior dislocation model where the vertebra is dislocated dorsally. The model attempts to closely reproduce the anterior fracture-dislocation injuries that are more commonly seen than later dislocations. The model is advantageous in that surgical exposure of the spinal cord is not required and the spinal column is intact. However, behavioral studies to characterize the model have not been performed and reproducibility of this model has yet to be proven.

\section{TRANSECTION MODELS}

Complete and partial transection models have been utilized extensively, particularly in tissue engineering, to investigate neuronal regeneration following injury (Figures $3 \mathrm{a}-\mathrm{c}$ ). They are beneficial for assessment of axonal regeneration and subsequent functional recovery. However, these models are less ideal for investigating the complex pathophysiology involved in SCI because spinal cord transections are not common in clinical settings.

\section{Full transection}

A full transection model encompasses a complete disassociation between the caudal and rostral segments and is advantageous in its easy reproducibility. Following spinal cord severance, a complex pathophysiological cascade ensues that inhibits potential axonal regrowth and forms scar tissue (Figures $3 \mathrm{~d}-\mathrm{g}$ ). ${ }^{38}$ It is also important to note that there is evidence to support native locomotive abilities in animals with a fully transected spinal cord because of inherent spinal cord circuitry. ${ }^{39}$ Thus, in order to assure that natural or intervention-based recovery is truly determined by the 
transection, the cord should be retransected to observe loss of regained function. ${ }^{40}$ Transection models, being relatively easy to perform, have been investigated in a variety of animals including rats, mice, cats, dogs and primates. ${ }^{41}$

\section{Partial transection}

Partial transection, unilateral transection and hemisection of the spinal cord all refer to the process of selectively lesioning the spinal cord (Figures $3 \mathrm{a}-\mathrm{c}$ ). These models simulate an injury more likely to be seen clinically than complete transection and provide for comparison between injured and healthy fibers in the same animal. For instance, hemisection may be used to examine locomotor function and recovery in different spinal tracts or to compare deficits in function when comparing contra- and ipsilateral lesions. The technique has also recently been used to investigate nerve grafting, a potentially promising surgical treatment for $\mathrm{SCI}^{42}$ In addition, given that hemisection results in a less severe injury than complete transection, postoperative animal care is easier. ${ }^{40}$ However, it is more difficult to inflict a consistent injury with a partial transection model, and it can be difficult to determine whether the target tract is completely severed. This concern can be addressed with retrograde labeling or, more recently, somatosensory evoked potentials. ${ }^{43}$

\section{CHEMICAL MODELS}

Numerous models have been developed that use chemicals to mimic a specific aspect of the secondary injury cascade that follows traumatic SCI. These models are useful for investigating molecular mechanisms involved in SCI and the effect of various therapies on specific pathways. However, chemical models are less useful than other models in terms of overall approximation to SCI. Other limitations relate to the delivery and reproducibility of exact location, extend and characterization of the injury produced.

Photochemically induced ischemia models involve injection or direct application of a nontoxic photosensitive dye, like rose bengal and eythrosin, followed by irradiation of the area to be injured for select duration of time. ${ }^{44}$ SCI models to approximate the excitotoxicity secondary pathway can be produced by injection of glutamate, aspartate, $\mathrm{N}$-methyl-D-aspartate or kainate (a glutamate receptor agonist) at desired spinal cord level using microdialysis technique. ${ }^{45}$ The administration of reagents, such as superoxide, hydroxyl radical and peroxynitrate, ${ }^{46}$ has been utilized to generate reactive oxygen species (for example, free radicals) that ultimately cause oxidative damage to lipids and proteins, thereby damaging neurons. Inflammatory SCI can be induced by injection of zymosan, a yeast particulate that activates macrophages and thereby causes neuroinflammation. Injection of phospholipase $A_{2}$ has also been shown to initiate inflammation and cell tissue damage that are characteristic of traumatic SCI. ${ }^{47}$

Chemical models of demyelination are useful for evaluation of therapies directed to enhance remyelination. These models include injection of ethidium bromide, lysolethicin, murine hepatitis virus, or a cocktail of myelin-specific antibodies and complement. Alternatively, Cuprizone can also be introduced in the animal's diet. In all cases, remyelination occurs spontaneously, but at different rates depending on the treatment. ${ }^{48}$ Syringomyelia, which is characterized by fluid-filled cavity formation, occurs in up to $20 \%$ of SCI cases.

Table 1 Summary of SCI models

\begin{tabular}{|c|c|c|c|c|}
\hline Model & Mechanism & Strengths & Weakness & Animal model \\
\hline MASCIS & Weight-drop contusion & Widely used, validated, reproducible, & $\begin{array}{l}\text { Procedure learning curve, 'Weight-bounce', } \\
\text { imprecise duration of impact }\end{array}$ & Rodents \\
\hline Infinite horizon & $\begin{array}{l}\text { Force-controlled } \\
\text { contusion }\end{array}$ & Widely used, validated, reproducible & Procedure learning curve, clamping technique & Rodents \\
\hline OSU & Displacement contusion & Validated, reproducible & Procedure learning curve & Rodents \\
\hline Air-gun impactor & Air-pressure contusion & Less invasive & $\begin{array}{l}\text { Not validated, inconsistent severity-based locomo- } \\
\text { tor assessment }\end{array}$ & Rats \\
\hline Clip & Compression-contusion & $\begin{array}{l}\text { Widely used, simple and easy procedure, } \\
\text { limited resources necessary, inexpensive }\end{array}$ & $\begin{array}{l}\text { Velocity of force, force delivered, extend of } \\
\text { compression not recorded }\end{array}$ & Murine, rats \\
\hline $\begin{array}{l}\text { Forceps } \\
\text { compression }\end{array}$ & Compression & $\begin{array}{l}\text { Simple, inexpensive, limited resources } \\
\text { necessary }\end{array}$ & $\begin{array}{l}\text { Lack acute injury aspect, inability to record injury } \\
\text { parameters }\end{array}$ & Pig, rat \\
\hline $\begin{array}{l}\text { Balloon } \\
\text { compression }\end{array}$ & Compression-contusion & Inexpensive & $\begin{array}{l}\text { Less common, injury parameters not recorded, } \\
\text { inconsistent injury }\end{array}$ & $\begin{array}{l}\text { Dogs, rat, rabbit, } \\
\text { primates }\end{array}$ \\
\hline $\begin{array}{l}\text { Spinal cord } \\
\text { strapping }\end{array}$ & Compression-contusion & Less-invasive, circumferential injury & $\begin{array}{l}\text { Not validated, inconsistent, injury parameters not } \\
\text { recorded }\end{array}$ & Rat \\
\hline $\begin{array}{l}\text { Harrington } \\
\text { distractor }\end{array}$ & Distraction & Clinical approximation & $\begin{array}{l}\text { Not validated, inconsistent and variable injury, } \\
\text { complex procedure }\end{array}$ & Rat \\
\hline UBC device & $\begin{array}{l}\text { Contusion-distraction- } \\
\text { dislocation }\end{array}$ & Clinical SCI approximation & $\begin{array}{l}\text { Not validated, inconsistent and variable injury, } \\
\text { complex procedure, no locomotor assessment }\end{array}$ & Rat \\
\hline UTA distractor & Distraction & Bidirectional distraction & $\begin{array}{l}\text { Not validated, inconsistent and variable injury, } \\
\text { complex procedure, no locomotor assessment }\end{array}$ & Rat \\
\hline $\begin{array}{l}\text { Complete } \\
\text { transection }\end{array}$ & Transection & $\begin{array}{l}\text { Easy to perform, consistent, reliable, large } \\
\text { animal models }\end{array}$ & Uncommon clinical SCI type & $\begin{array}{l}\text { Rodents, cats, dogs, } \\
\text { primates }\end{array}$ \\
\hline $\begin{array}{l}\text { Partial } \\
\text { transection }\end{array}$ & Transection & $\begin{array}{l}\text { Large animal models, contralateral side } \\
\text { comparison }\end{array}$ & Inconsistent reproducibility & $\begin{array}{l}\text { Rodents, cats, dogs, } \\
\text { primates }\end{array}$ \\
\hline $\begin{array}{l}\text { Chemical } \\
\text { models }\end{array}$ & Reagents & $\begin{array}{l}\text { Inexpensive, simple procedure, mechanism } \\
\text { specific }\end{array}$ & Inconsistent reproducibility & Rodents \\
\hline
\end{tabular}


Chemical technique to recreate this condition has been developed in rats and involves injection of a kaolin suspension or quisqualic acid at different sites with the spinal cord, including the parenchyma and central gray matter. ${ }^{49}$

\section{CONCLUSION}

Given the complexity of human SCI, no one model can encompass all aspects of injury. Each model aims to generate a consistent, easily reproducible and graded injury that mirrors SCI pathology in humans as closely as possible. Substantial progress has been made in the field over the past 20 years for improvement and refinement of existing models and the development of novel new paradigms. The general strengths and weakness of current SCI models have been summarized in Table 1. Correlation between amount of axonal survival and maintenance of motor function after injury depends on injury model, species and method of behavioral assessment. ${ }^{50}$ Although newer SCI models aim to better approximate human SCI in various aspects, these models require further improvement and validation. The optimal choice of model for a given study depends on the study goals, specific parameters and resources of the investigation.

\section{DATA ARCHIVING}

There were no data to deposit.

\section{CONFLICT OF INTEREST}

The authors declare no conflict of interest.

1 Allen AR. Surgery of experimental lesion of spinal cord equivalent to crush injury of fracture dislocation of spinal column a preliminary report. J Am Med Assoc 1911; 57 878-880.

2 Metz GAS, CURT A, van de MEENT H, KLUSMAN I, SCHWAB ME, DIETZ V. Validation of the weight-drop contusion model in rats: a comparative study of human spinal cord injury. J Neurotrauma 2000; 17: 1-17.

3 Jakeman LB, Guan Z, Wei P, Ponnappan R, Dzwonczyk R, Popovich PG et al. Traumatic spinal cord injury produced by controlled contusion in mouse. J Neurotrauma 2000; 17: 299-319.

4 Iwanami A, Yamane J, Katoh H, Nakamura M, Momoshima S, Ishii $\mathrm{H}$ et al. Establishment of graded spinal cord injury model in a nonhuman primate: the common marmoset. J Neurosci Res 2005; 80: 172-181.

5 Dunham KA, Siriphorn A, Chompoopong S, Floyd CL. Characterization of a graded cervical hemicontusion spinal cord injury model in adult male rats. J Neurotrauma 2010; 27: 2091-2106

6 Magnuson DS, Trinder TC, Zhang YP, Burke D, Morassutti DJ, Shields CB. Comparing deficits following excitotoxic and contusion injuries in the thoracic and lumbar spinal cord of the adult rat. Exp Neurol 1999; 156: 191-204.

7 Gruner JA. A monitored contusion model of spinal cord injury in the rat. J Neurotrauma 1992; 9: 123-128.

8 Pan JZ, Ni L, Sodhi A, Aguanno A, Young W, Hart RP. Cytokine activity contributes to induction of inflammatory cytokine mRNAs in spinal cord following contusion. J Neurosci Res 2002; 68: 315-322.

9 Hu R, Zhou J, Luo C, Lin J, Wang X, Li X et al. Glial scar and neuroregeneration: histological, functional, and magnetic resonance imaging analysis in chronic spinal cord injury. J Neurosurg Spine 2010; 13: 169-180.

10 Jones CF, Lee JH, Kwon BK, Cripton PA. Development of a large-animal model to measure dynamic cerebrospinal fluid pressure during spinal cord injury: Laboratory investigation. J Neurosurg Spine 2012; 16: 624-635.

11 Scheff SW, Rabchevsky AG, Fugaccia I, Main JA, Lumpp JE Jr. Experimental modeling of spinal cord injury: characterization of a force-defined injury device. J Neurotrauma 2003; 20: 179-193.

12 Bottai D, Cigognini D, Madaschi L, Adami R, Nicora E, Menarini M et al. Embryonic stem cells promote motor recovery and affect inflammatory cell infiltration in spinal cord injured mice. Exp Neurol 2010; 223: 452-463.

13 Streijger F, Plunet WT, Lee JH, Liu J, Lam CK, Park S et al. Ketogenic diet improves forelimb motor function after spinal cord injury in rodents. PLOS ONE 2013; 8 e78765.

14 Stokes BT. Experimental spinal cord injury: a dynamic and verifiable injury device. J Neurotrauma 1992; 9: 129-134.

15 Pearse D, Lo T Jr, Cho K, Lynch M, Garg M, Marcillo A et al. Histopathological and behavioral characterization of a novel cervical spinal cord displacement contusion injury in the rat. J Neurotrauma 2005; 22: 680-702.
16 Marcol W, Slusarczyk W, Gzik M, Larysz-Brysz M, Bobrowski M, Grynkiewicz-Bylina B et al. Air gun impactor-a novel model of graded white matter spinal cord injury in rodents. J Reconstr Microsurg 2012; 28: 561-568.

17 Rivlin A, Tator C. Effect of duration of acute spinal cord compression in a new acute cord injury model in the rat. Surg Neurol 1978; 10: 38 .

18 Poon PC, Gupta D, Shoichet MS, Tator CH. Clip compression model is useful for thoracic spinal cord injuries: histologic and functional correlates. Spine 2007; 32: 2853-2859.

19 Steward O, Schauwecker PE, Guth L, Zhang Z, Fujiki M, Inman D et al. Genetic approaches to neurotrauma research: opportunities and potential pitfalls of murine models. Exp Neurol 1999; 157: 19-42.

20 Joshi M, Fehlings MG. Development and characterization of a novel, graded model of clip compressive spinal cord injury in the mouse: Part 1. Clip design, behavioral outcomes, and histopathology. J Neurotrauma 2002; 19: 175-190.

21 Marques SA, Garcez VF, Del Bel EA, Martinez AMB. A simple, inexpensive and easily reproducible model of spinal cord injury in mice: morphological and functional assessment. J Neurosci Methods 2009; 177: 183-193.

22 Skinner SA, Transfeldt EE. Electromyography in the detection of mechanically induced spinal motor tract injury: observations in diverse porcine models. J Neurosurg Spine 2009; 11: 369-374.

23 Zivin JA, DeGirolami U. Spinal cord infarction: a highly reproducible stroke model. Stroke 1980; 11: 200-202.

24 Coston A, Laville M, Baud P, Bussel B, Jalfre M. Aortic occlusion by a balloon catheter: a method to induce hind limb rigidity in rats. Physiol Behav 1983; 30: 967-969.

25 Blight AR. Morphometric analysis of a model of spinal cord injury in guinea pigs, with behavioral evidence of delayed secondary pathology. J Neurol Sci 1991; 103 $156-171$.

26 Plemel JR, Duncan G, Chen KWK, Shannon C, Park S, Sparling JS et al. A graded forceps crush spinal cord injury model in mice. J Neurotrauma 2008; 25: 350-370.

27 Tarlov I, Klinger $\mathrm{H}$, Vitale S. Spinal cord compression studies: I. experimental techniques to produce acute and gradual compression. Arch Neurol Psychiatry 1953; 70: 813

28 Vanický I, Urdzíková L, Saganová K, Cízková D, Gálik J. A simple and reproducible model of spinal cord injury induced by epidural balloon inflation in the rat. J Neurotrauma 2001; 18: 1399-1407.

29 Aslan A, Cemek M, Eser O, Altunbaş K, Buyukokuroglu ME, Cosar M et al. Does dexmedetomidine reduce secondary damage after spinal cord injury? An experimental study. Eur Spine J 2009; 18: 336-344.

30 Fukuda S, Nakamura T, Kishigami Y, Endo K, Azuma T, Fujikawa T et al. New canine spinal cord injury model free from laminectomy. Brain Res Protoc 2005; 14 $171-180$

31 Nesathurai S, Graham WA, Mansfield K, Magill D, Sehgal P, Westmoreland SV et al Model of traumatic spinal cord injury in Macaca fascicularis: similarity of experimental lesions created by epidural catheter to human spinal cord injury. J Med Primatol 2006; 35: 401-404.

32 da Costa ESA, Carvalho AL, Martinez AMB, De-Ary-Pires B, Pires-Neto MA de Ary-Pires R. Strapping the spinal cord: an innovative experimental model of CNS injury in rats. J Neurosci Methods 2008; 170: 130-139.

33 Silberstein M, McLean K. Non-contiguous spinal injury: clinical and imaging features, and postulated mechanism. Spinal Cord 1994; 32: 817-823.

34 Dabney KW, Ehrenshteyn M, Agresta CA, Twiss JL, Stern G, Tice L et al. A mode of experimental spinal cord trauma based on computer-controlled intervertebral distraction: characterization of graded injury. Spine 2004; 29: 2357.

35 Choo AMT, Liu J, Liu Z, Dvorak M, Tetzlaff W, Oxland TR. Modeling spinal cord contusion, dislocation, and distraction: characterization of vertebral clamps, injury severities, and node of Ranvier deformations. J Neurosci Methods 2009; 181: 6-17.

36 Seifert J, Bell J, Elmer B, Sucato D, Romero M. Characterization of a nove bidirectional distraction spinal cord injury animal model. J Neurosci Methods 2011, 197: 97-103.

37 Fiford RJ, Bilston LE, Waite P, Lu J. A vertebral dislocation model of spinal cord injury in rats. J Neurotrauma 2004; 21: 451-458.

38 Spillmann AA, Amberger VR, Schwab ME. High molecular weight protein of human central nervous system myelin inhibits neurite outgrowth: an effect which can be neutralized by the monoclonal antibody IN-1. Eur J Neurosci 2006; 9 549-555.

39 Barbeau H, McCrea DA, O'Donovan MJ, Rossignol S, Grill WM, Lemay MA. Tapping into spinal circuits to restore motor function. Brain Res Rev 1999; 30: 27-51.

40 Kwon BK, Oxland TR, Tetzlaff W. Animal models used in spinal cord regeneration research. Spine 2002; 27: 1504-1510.

41 Heimburger R. Return of function after spinal cord transection. Spinal Cord 2005; 43 438-440.

42 Alilain WJ, Horn KP, Hu H, Dick TE, Silver J. Functional regeneration of respiratory pathways after spinal cord injury. Nature 2011; 475: 196-200.

43 Cloud BA, Ball BG, Chen B, Knight AM, Hakim JS, Ortiz AM et al. Hemisection spinal cord injury in rat: the value of intraoperative somatosensory evoked potential monitoring. J Neurosci Methods 2012; 211: 179-184.

44 Watson BD, Prado R, Dalton Dietrich W, Ginsberg MD, Green BA. Photochemically induced spinal cord injury in the rat. Brain Res 1986; 367: 296-300.

45 Liu D, Xu GY, Pan E, McAdoo D. Neurotoxicity of glutamate at the concentration released upon spinal cord injury. Neuroscience 1999; 93: 1383-1389.

46 Bao F, DeWitt DS, Prough DS, Liu D. Peroxynitrite generated in the rat spinal cord induces oxidation and nitration of proteins: Reduction by $\mathrm{Mn}$ (III) tetrakis (4-benzoic acid) porphyrin. J Neurosci Res 2002; 71: 220-227. 
47 Liu NK, Zhang YP, Titsworth WL, Jiang X, Han S, Lu PH et al. A novel role of phospholipase A2 in mediating spinal cord secondary injury. Ann Neurol 2006; 59: 606-619.

48 Jernigan SC, Zhang YP, Shields CB, Whittemore SR. Rodent spinal cord demyelination models. In: Chen J, Xu X and Xu Z (eds) Animal Models of Acute Neurological Injuries. Humana Press: Totowa, NJ, 2009, pp 471-478.
49 Lee GYF, Jones NR, Mayrhofer G, Brown C, Cleland L. Origin of macrophages in a kaolin-induced model of rat syringomyelia: a study using radiation bone marrow chimeras. Spine 2005; 30: 194-200.

50 Fehlings MG, Tator $\mathrm{CH}$. The relationships among the severity of spinal cord injury, residual neurological function, axon counts, and counts of retrogradely labeled neurons after experimental spinal cord injury. Exp Neurol 1995; 132: 220-228. 